https://doi.org/10.15407/ujpe64.3.189

O.S. STASHKO, V.I. ZHDANOV

Taras Shevchenko National University of Kyiv, Ukraine

(60, Volodymyrs'ka Str., Kyiv 01601, Ukraine; e-mail: alexander.stashko@gmail.com)

\title{
SPHERICALLY SYMMETRIC CONFIGURATIONS IN GENERAL RELATIVITY IN THE PRESENCE OF A LINEAR MASSIVE SCALAR FIELD: SEPARATION OF A DISTRIBUTION OF TEST BODY CIRCULAR ORBITS
}

\begin{abstract}
We study static spherically symmetric configurations in the presence of linear massive scalar fields within General Relativity. Static solutions of the Einstein equations are considered under conditions of asymptotic flatness. Each solution is fixed by the configuration mass and the field strength parameter, which are defined at spatial infinity. The metric coefficients and the scalar field for a specific configuration are obtained numerically. Then we study the time-like geodesics describing the test particle motion. The focus is on the distribution of stable circular orbits (SCOs) of the test particles around a configuration. We found that, for the continuum of configuration parameters, there exist two unlinked regions of SCOs that are separated by some annular region, where SCOs do not exist.
\end{abstract}

K e y w ords: relativistic astrophysical objects, scalar fields, accretion disks.

\section{Introduction}

Theories with scalar fields are widely discussed in gravitational physics [1-4], when the scalar field (SF) acts as an independent physical entity or as an auxiliary tool that helps to highlight merits of general relativity in experimental tests and to understand some properties of more complicated structures (with higher spins, etc.). In particular, it is widely assumed that some form of a dynamical dark energy (DE) provided the inflation of the very early Universe [2], and the theories with scalar fields (SFs) occupy an important sector of this area (see, e.g., [5,6]). The abundance of various cosmological models draws attention to unifying the schemes and interrelations between competing dark energy candidates [3, 4, 7], in particular, between scalar field models and the traditional hydrodynamical approach in cosmology (see, e.g., [8-13]).

On the other hand, one can look for effects of SFs in relativistic compact astrophysical objects. It should be noted that, for a wide class of nonlinear wave equations, there exist no regular stable stationary solutions [14]. Therefore, a solution with SF must have some kind of singularity. There is an analytic solution

(c) O.S. STASHKO, V.I. ZHDANOV, 2019

ISSN 2071-0194. Ukr. J. Phys. 2019. Vol. 64, No. 3 with the massless SF that is singular at the origin $[15,16]$; this solution shows that an arbitrarily small SF strongly affects the space-time topology leading to the naked singularity. Particular numerical solutions for a massive scalar field were found by Asanov [19], also with the singularity. On the other hand, Bekenstein ([17]; see also [18] and references therein) showed that a stationary black hole cannot be endowed with exterior SF.

Different DE models can manifest themselves through the geodesic structure of the space-time near compact astrophysical objects. In particular, the distribution of test body circular orbits may give us a hint about real accretion disks around these objects (see, e.g., [21-28] and references therein). Examples show that, in a number of cases, the disconnected structures of stable circular orbits (SCOs) can arise [25-31]. In our previous papers [29-31], this question was considered for specially chosen SF potentials that enabled us to perform a semianalytic treatment. In particular, we showed that the discontinuous distributions of SCOs can arise both in the case of a spacetime with naked singularities and in the black hole space times.

In the presence of a linear massless real SF, the existence of disconnected regions of SCOs separated by 
a ring of unstable orbits was shown in [23], by using an analytic solution $[15,16]$. In the present paper, we extend this result to the massive case with the potential $V(\phi)=\mu_{f}^{2} \phi^{2} / 2$. Note that, in the previous paper [30], we considered the massive case for artificially chosen SF potentials. Because of the absence of an exact analytic solution of the Einstein-SF equations, we treat the problem numerically. We will derive asymptotic relations for the metric and SF at the spatial infinity (Section 3) and then continue the solutions numerically from large values of a radial variable to smaller ones (Section 4). The distribution of SCOs is studied in Section 5, by using the effective potential for test body trajectories.

\section{General Relations and Notations}

We consider a configuration with massive linear scalar field (SF) $\phi$ defined by the Lagrangian density

$L=\frac{1}{2} \phi,{ }_{\mu} \phi^{, \mu}-\frac{\mu_{f}^{2}}{2} \phi^{2}, \quad \mu_{f}>0$.

The space-time with metric $d s^{2}=g_{\mu \nu} d x^{\mu} d x^{\nu}$ obeys the Einstein equations

$G_{\mu}^{\nu}=8 \pi T_{\mu}^{\nu}$

and is assumed to be asymptotically flat. In this paper, we use units, in which $c=G=1$.

We deal with a static spherically symmetric spacetime. The coordinate system is fixed by the following expression for the metric:

$d s^{2}=e^{\alpha(r)} d t^{2}-e^{\beta(r)} d r^{2}-r^{2} d O^{2}$.

Here, $r \geq 0$, and $d O^{2}=d \theta^{2}+(\sin \theta)^{2} d \varphi^{2}$ is the interval on the unit sphere. The energy-momentum tensor of the field is

$T_{\mu \nu}=\phi_{, \mu} \phi_{, \nu}-g_{\mu \nu} L$.

The field equation

$g^{\mu \nu} \nabla_{\mu} \nabla_{\nu} \phi=-\mu_{f}^{2} \phi$

follows either from the covariant conservation law for (4), which is also the integrability condition of (2) with the energy-momentum tensor (4), or directly from the Lagrange equations for $L$.

Einstein's equations (2) for

$(\mu, \nu)=(0,0),(1,1),(2,2)$ yield three nontrivial equations for $\alpha(r), \beta(r)$, and $\phi(r)$ in the case of static metric (3) (see, e.g., [32]). We use two equations $(2)$ for $(\mu, \nu)=(0,0)$ and $(\mu, \nu)=(1,1)$, which can be written as

$\frac{d}{d r}\left[r\left(e^{-\beta}-1\right)\right]=-8 \pi r^{2} T_{0}^{0}$,

$r e^{-\beta} \frac{d \alpha}{d r}+e^{-\beta}-1=-8 \pi r^{2} T_{1}^{1}$.

In the case of static metric (3), Eq. (5) yields

$\frac{d}{d r}\left[r^{2} e^{\frac{\alpha-\beta}{2}} \frac{d \phi}{d r}\right]=r^{2} e^{\frac{\alpha+\beta}{2}} \mu_{f}^{2} \phi$.

Equation $(2)$ for $(\mu, \nu)=(2,2)$ and the equivalent one for $(\mu, \nu)=(3,3)$ follow from $(6),(7)$, and (8).

\section{Boundary Conditions} and Asymptotic Behavior

We are looking for the functions $\alpha(r), \beta(r) \in$ $\in C^{1}(0, \infty)$, and $\phi(r) \in C^{2}(0, \infty)$, which satisfy (6) $-(8)$.

In view of the asymptotic flatness, we assume

$\lim _{r \rightarrow \infty}\left[r\left(e^{\alpha}-1\right)\right]=\lim _{r \rightarrow \infty}\left[r\left(e^{-\beta}-1\right)\right]=-r_{g}$,

where $r_{g}=2 M$, and $M>0$ is the configuration mass. It is also assumed that the field $\phi(r)$ and its derivative tend to zero sufficiently rapidly at the spatial infinity similarly to the flat space case where $\phi(r) \sim \exp \left(-\mu_{f} r\right)$ (to within a power-law factor).

Multiplying (8) by $\phi$ yields

$\frac{d}{d r}\left[r^{2} e^{\frac{\alpha-\beta}{2}} \phi \frac{d \phi}{d r}\right]=$

$=r^{2} e^{\frac{\alpha-\beta}{2}}\left[\frac{d \phi}{d r}\right]^{2}+\mu_{f}^{2} r^{2} e^{\frac{\alpha+\beta}{2}} \phi^{2}$.

Given the assumption about the behavior at infinity

$\lim _{r \rightarrow \infty}\left[r^{2} \frac{d \phi^{2}}{d r}\right]=0$

and taking (9) into account, we have

$\lim _{r \rightarrow \infty}\left[r^{2} e^{\frac{\alpha-\beta}{2}} \phi \frac{d \phi}{d r}\right]=0$.

Then the integration of (10) yields

$-r^{2} e^{\frac{\alpha-\beta}{2}} \phi \frac{d \phi}{d r}=$

ISSN 2071-0194. Ukr. J. Phys. 2019. Vol. 64, No. 3 
$=\int_{r}^{\infty} x^{2} e^{\alpha(x) / 2}\left\{e^{-\beta(x) / 2}\left[\frac{d \phi}{d x}\right]^{2}+\mu_{f}^{2} e^{\beta(x) / 2} \phi(x)^{2}\right\} d x$.

This means that, in the case of regular $\alpha(r), \beta(r)$, and $\phi(r)$ and under condition (11), the non-trivial $\phi(r)$, as well as $d \phi^{2} / d r<0$, cannot be zero for any $r$ on $(0, \infty)$, i.e. these functions do not change their signs, and $|\phi(r)|$ is a monotonically decreasing function. Moreover, $\exp [(\alpha-\beta) / 2] d \phi^{2} / d r \rightarrow \infty$ for $r \rightarrow 0$ as $r^{-2}$ or faster, and at least one of the functions $\exp [(\alpha-\beta) / 2]$ and $d \phi^{2} / d r$ must be singular as $r \rightarrow 0$.

For definiteness, we further assume that $\phi(r)>0$ and, correspondingly, $d \phi / d r<0$.

In the case of Lagrangian (1) for the energymomentum (4), we have $T_{0}^{0}=e^{-\beta} \phi^{\prime 2} / 2+\mu_{f}^{2} \phi^{2} / 2$ and $T_{1}^{1}=-e^{-\beta} \phi^{\prime 2} / 2+\mu_{f}^{2} \phi^{2} / 2$. Equations (6-8) form a complete system for the numerical integration under the assumptions concerning the asymptotic behavior at the spatial infinity. Further, we integrate this system backward, by starting from sufficiently large values of $r$. This needs approximate analytic solutions for large $r$. To facilitate the analytic calculations, it is convenient to introduce a formal parameter $\varepsilon$ into (6) and (7):

$\frac{d}{d r}\left[r\left(e^{-\beta}-1\right)\right]=-4 \varepsilon \pi r^{2}\left(e^{-\beta} \phi^{2}+\mu_{f}^{2} \phi^{2}\right)$,

$r e^{-\beta} \frac{d \alpha}{d r}+e^{-\beta}-1=-4 \varepsilon \pi r^{2}\left(-e^{-\beta} \phi^{2}+\mu_{f}^{2} \phi^{2}\right)$.

We set

$e^{-\beta}=1-\frac{r_{g}}{r}-\varepsilon \beta_{*}(r), \quad e^{\alpha}=1-\frac{r_{g}}{r}+\varepsilon \alpha_{*}(r)$.

Then we can look for the solutions as a formal expansion in powers of $\varepsilon$, by setting $\varepsilon=1$ in the final results. This is the same as an expansion in powers of the gravitational constant (see, e.g., [20]).

In view of (15), we rewrite (13) as

$\frac{d}{d r}\left(r \beta_{*}\right)=4 \pi r^{2}\left(e^{-\beta} \phi^{2}+\mu_{f}^{2} \phi^{2}\right)$.

Substituting the lowest approximation $(\varepsilon=0)$ of (15) into Eq. (8), we get

$\phi(r)=Q \frac{e^{-\mu_{f} r}}{r^{1+\kappa}} \psi_{0}(r)$,

where $Q$ is an arbitrary constant that characterizes the field strength at the spatial infinity, $\kappa=\mu_{f} r_{g} / 2$,

ISSN 2071-0194. Ukr. J. Phys. 2019. Vol. 64, No. 3 and $\psi_{0}(r)$ is the unique solution of the equation

$e^{\mu_{f} r} \frac{d}{d r}\left[r^{2}\left(1-\frac{r_{g}}{r}\right) \frac{d}{d r}\left(\frac{e^{-\mu_{f} r}}{r^{1+\kappa}} \psi_{0}(r)\right)\right]=\mu_{f}^{2} r^{2} \frac{\psi_{0}(r)}{r^{1+\kappa}}$

and satisfies $\psi_{0}(\infty)=1$. The parameters $M=r_{g} / 2$ and $Q$ completely characterize the solution and the whole spherically symmetric configuration. The solution $\psi_{0}(r)$ of (18) can be found in the form of an asymptotic series in powers of $r^{-1}$.

Then we proceed to (14), (16). Discarding the terms $\sim \varepsilon^{2}$ and higher orders in $\varepsilon$ in these equations, we have

$\beta_{*}(r)=-4 \pi Q^{2} \mu_{f} \frac{e^{-2 \mu_{f} r}}{r^{2 \kappa+1}}\left[1+O\left(\frac{1}{r}\right)\right]$,
$\alpha_{*}(r)=-2 \pi Q^{2} \frac{e^{-2 \mu_{f} r}}{r^{(2 \kappa+2)}}\left[1+O\left(\frac{1}{r}\right)\right]$.

Further approximations show that the asymptotic solution can be represented in the form

$\phi(r)=Q \frac{e^{-\mu_{f} r}}{r^{1+\kappa}} \sum_{n=0}^{N} \varepsilon^{n} \frac{e^{-2 n \mu_{f} r}}{r^{n(2 \kappa+1)}} \psi_{n}(r)+\ldots$

$\alpha_{*}(r)=\sum_{n=1}^{N} \varepsilon^{n-1} \frac{e^{-2 n \mu_{f} r}}{r^{n(2 \kappa+1)}} \alpha_{n}(r)+\ldots$,

$\beta_{*}(r)=\sum_{n=1}^{N} \varepsilon^{n-1} \frac{e^{-2 n \mu_{f} r}}{r^{n(2 \kappa+1)}} \beta_{n}(r)+\ldots$,

where $\psi_{n}, \alpha_{n}$, and $\beta_{n}$ can be expanded, in turn, in powers of $1 / r$ as follows:

$\psi_{n}(r)=\sum_{m=0}^{M} \frac{A_{n, m}}{r^{m}}+\ldots$

$\alpha_{n}(r)=\sum_{m=0}^{M} \frac{a_{n, m}}{r^{m}}+\ldots, \quad \beta_{n}(r)=\sum_{m=0}^{M} \frac{b_{n, m}}{r^{m}}+\ldots ;$

$A_{0,0}=1$, and the other coefficients $A_{n, m}, a_{n, m}, b_{n, m}$ can be obtained in the unique way, by equating terms with equal powers in $\varepsilon$, $\exp \left(-\mu_{f} r\right)$, and $1 / r$ on the both sides of (8), (14), (16); $M, N$ are defined by the prescribed accuracy of the approximation.

For example, the first coefficients are $\left(\xi=\mu_{f} r_{g}\right)$

$A_{0,0}=1, \quad A_{0,1}=\frac{r_{g}}{8}(2+3 \xi)$, 


$$
\begin{aligned}
& A_{0,2}=r_{g}^{2}\left(\frac{1}{8 \xi}+\frac{5}{32}+\frac{\xi}{4}+\frac{9 \xi^{2}}{128}\right) \\
& a_{1,0}=0, \quad a_{1,1}=-2 \pi Q^{2}, \\
& a_{0,2}=2 \pi Q^{2}\left(\frac{1}{\xi}+1-\frac{3}{4} \xi\right) \\
& b_{1,0}=-4 \pi Q^{2} \mu_{f}, \quad b_{1,1}=-\pi Q^{2}\left(4+3 \xi^{2}\right), \\
& b_{1,2}=-\pi Q^{2} r_{g} \xi\left(3+\frac{5}{4} \xi+\frac{9}{8} \xi^{2}\right) .
\end{aligned}
$$

The coefficients are generally non-analytic in $\mu_{f}$, and the higher orders of the expansion contain even higher orders of $\mu_{f}^{-1}$.

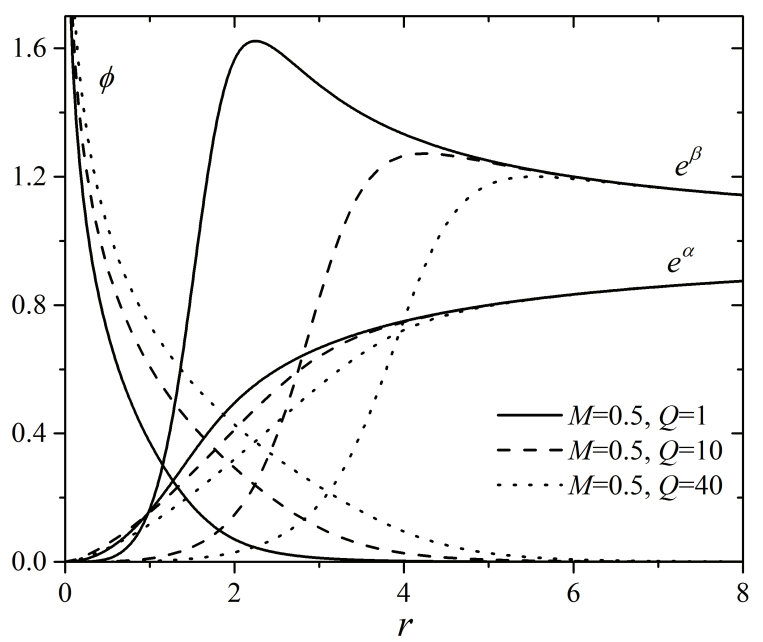

Fig. 1. $\left.g_{00}=e^{\alpha(r)}, g_{11}=-e^{\beta(r)}\right)$ and the field $\phi(r)$ for different $Q, m$ fixed

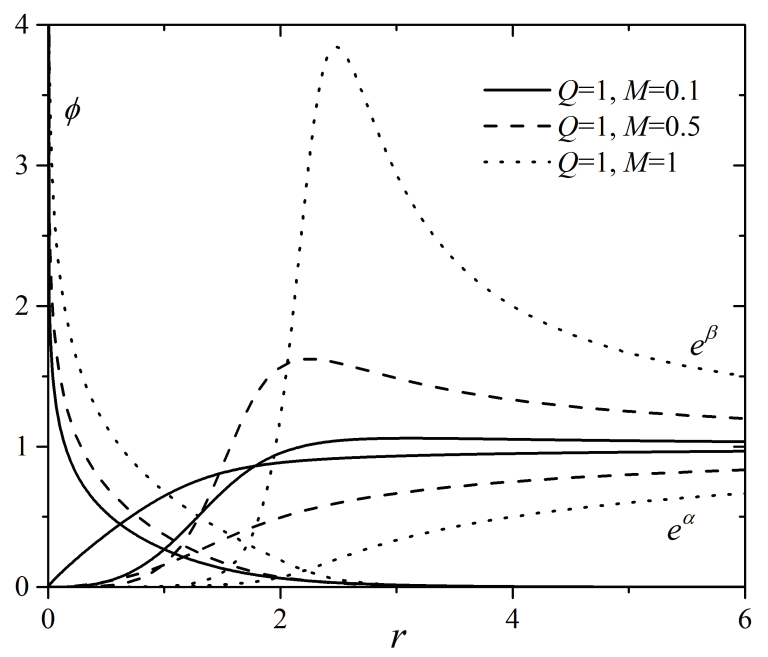

Fig. 2. The same for different $m, Q$ fixed; $e^{\alpha(r)}$ goes lower than $e^{\beta(r)}$ for larger $r$

192

\section{Numerical Solutions}

We used the asymptotic relations (21)-(23) with $\varepsilon=$ $=1$ to derive initial conditions $\phi\left(x_{\mathrm{inf}}\right), \phi^{\prime}\left(x_{\mathrm{inf}}\right)$, $\alpha\left(x_{\text {inf }}\right), \quad \beta\left(x_{\text {inf }}\right)$, which have been evaluated at a remote point $r=x_{\text {inf }} \gg r_{g}$. Then we integrate Eqs. (6)-(8) backward from $x_{\text {inf }}$ to the origin by means of the 4-th order Runge-Kutta method. As a result, we obtain a three-parameter family $\left(Q, M, \mu_{f}\right)$ of the asymptotically flat solutions.

The choice of $x_{\text {inf }}$ is a result of the compromise between the accuracy of the initial conditions and the number of terms in the asymptotic expansion. We rescaled the parameters $r$ and $M$ to $r \mu_{f}$ and $M \mu_{f}$, which corresponds to measuring $r$ and $M$ in the units of $1 / \mu_{f}$. After the rescaling, the typical choice of the initial point was $x_{\mathrm{inf}}=100$. In our stability tests of the numerical solutions, the computer experiments involved up to 30 terms of the expansion in $1 / r$.

Typical examples of the metric coefficients $g_{00}=e^{\alpha}$ and $g_{11}=-e^{\beta}$ and the scalar field $\phi$ as functions of $r$ are presented in Figs. 1 and 2. For all values of the parameters, we obtained $\exp [\alpha(r)] \rightarrow 0, \exp [\beta(r)] \rightarrow 0$, and $\exp [\alpha(r)]>\exp [\beta(r)]$ for smaller $r \rightarrow 0$. However, the sign of the latter inequality changes, as $r$ grows, and $\exp [\alpha(r)]<\exp [\beta(r)]$ for sufficiently large $r$. The field $\phi(r) \rightarrow \infty$ as $r \rightarrow 0$ and monotonically decreases to zero as $r \rightarrow \infty$.

\section{Distribution of Circular Orbits}

Here, we consider the time-like equatorial geodesics $(\theta=\pi / 2)$ of neutral test particles described by the integrals of motion

$e^{\alpha(r)}\left(\frac{d t}{d \tau}\right)=p_{t}, \quad r^{2}\left(\frac{d \varphi}{d \tau}\right)=L, \quad \theta=\pi / 2$,

where $\tau$ stands for the proper time of a particle.

These equations together with the normalization integral for the time-like geodesics yield the equation for the radial variable

$$
\begin{aligned}
& e^{\alpha+\beta}\left(\frac{d r}{d \tau}\right)^{2}=p_{t}^{2}-U_{\mathrm{eff}}(r, L), \\
& U_{\mathrm{eff}}(r, L)=e^{\alpha}\left(1+\frac{L^{2}}{r^{2}}\right) .
\end{aligned}
$$

Equation (26) formally describes a one-dimensional particle motion in a field with effective potential $U_{\text {eff }}$ Possible SCOs correspond to the points of minimum $r_{\text {min }}$ of $U_{\text {eff }}$, i.e., $U_{\text {eff }}^{\prime}(r)$ must change its sign from positive to negative, when passing $r_{\min }$.

ISSN 2071-0194. Ukr. J. Phys. 2019. Vol. 64, No. 3 


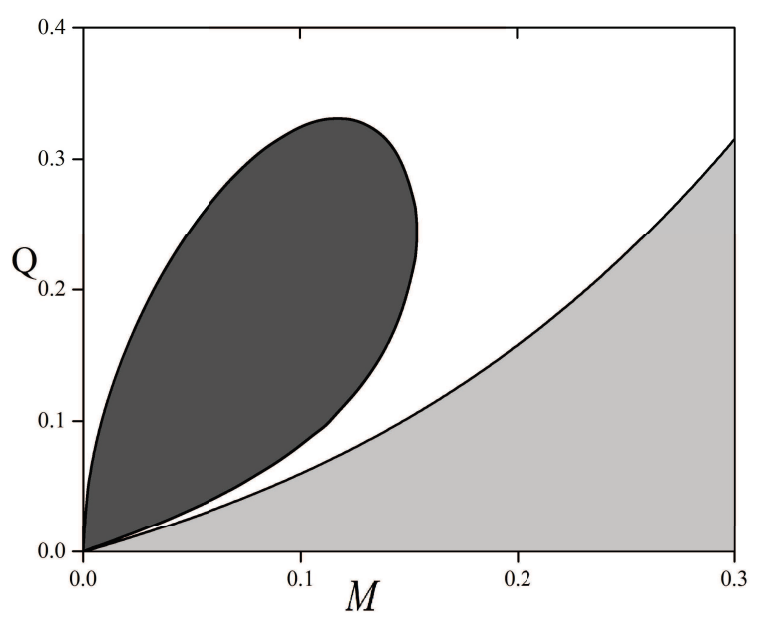

Fig. 3. Areas of parameters that are bounded by bifurcation curves, which represent configurations with qualitatively different distributions of SCOs. White region represents the parameters $(Q, M)$, for which the separated ring-like regions of SCOs exist. The dark grey region: there exist only stable SCOs for all non-zero values of angular momentum. Light grey region: the Schwarzschild-like case, there is an outer region of SCOs and a ring of unstable orbits

To study the distribution of SCOs, we used the method in [31], which is modified to the case of coordinates corresponding to (3).

The behavior of $U_{\text {eff }}$ is defined by the derivative

$\frac{d U_{\mathrm{eff}}}{d r}=e^{\alpha}\left[\alpha^{\prime}+\frac{L^{2}}{r^{3}}\left(r \alpha^{\prime}-2\right)\right]$.

If $r \alpha^{\prime}>2$, then $d U_{\text {eff }} / d r>0$, and there is no minima on this interval. If $r \alpha^{\prime}<2$, then the inequality $d U_{\text {eff }} / d r \lessgtr 0$ is equivalent to $F(r) \lessgtr L^{2}$, where we introduced the function

$F(r)=\frac{r^{3} \alpha^{\prime}(r)}{2-r \alpha^{\prime}(r)}$

Therefore, there is a local minimum $U_{\text {eff }}\left(r_{\text {min }}\right)$ if and only if

$F\left(r_{\min }\right)=L^{2}$,

and the function $F(r)$ is monotonically increasing in the neighborhood of $r_{\min }$.

Function (28) is useful to study possible minima and maxima of $U_{\text {eff }}(r, L)$ and their relative disposition. For example, we see in Fig. 5 that there are no configurations such that $U_{\text {eff }}$ has two minima, as there is no more than one solution of (29) on the interval, where $F(r)>0$ is monotonically increasing.

ISSN 2071-0194. Ukr. J. Phys. 2019. Vol. 64, No. 3
On the other hand, in the case of Fig. 4, this situation is possible, when two minima of $U_{\text {eff }}$ correspond to the same value of $L^{2}$. For fixed $Q, M$, from Fig. 4, we see that if $L^{2}$ changes, the radii of SCOs of the inner ring vary from zero to some $r_{1}$, which is the point of a maximum of $F(r)$ so that $F(r)$ is monotonically increasing on $\left(0, r_{1}\right)$. The radii of the outer ring vary from $r_{2}$ to infinity, where $r_{2}$ is the point of a minimum of $F(r)$.

Using $F(r) \equiv F(r, Q, M)$ that also depends on $(Q, M)$, we can find the configurations, where the

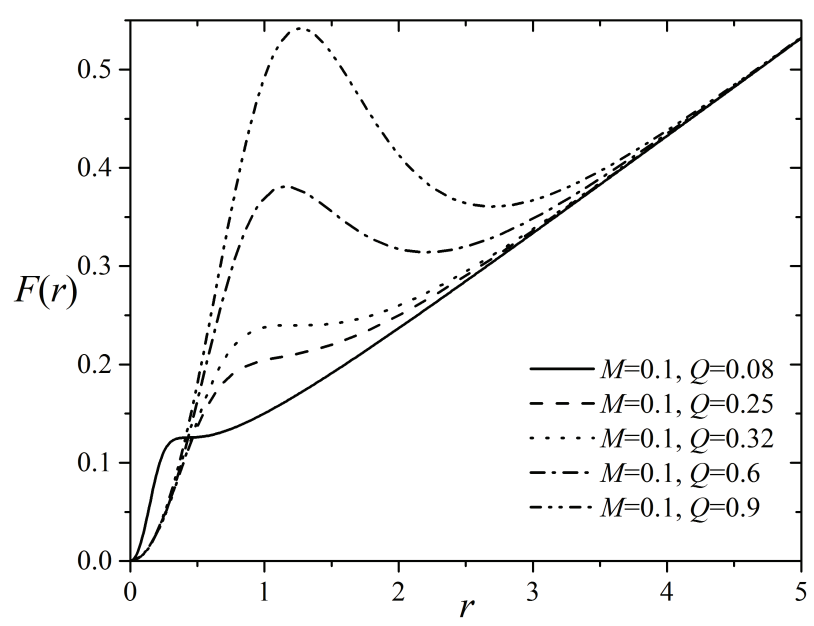

Fig. 4. Examples of $F(r)$ for configurations with different $Q, M=0.1$ corresponding to the dark grey (two lower curves) and white region (two upper curves) of Fig. 3. Dotted line describes an intermediate case

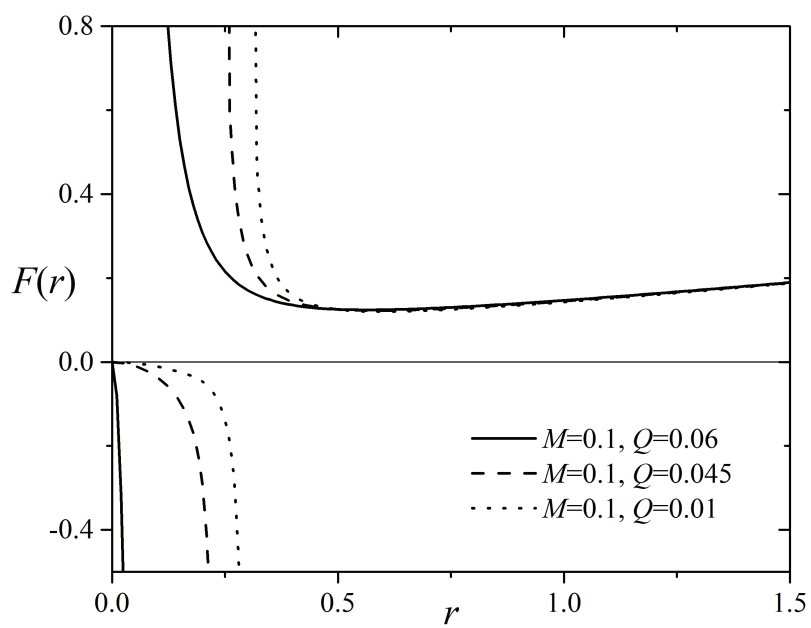

Fig. 5. Examples of $F(r)$ for configurations with different $Q, M=0.1$ corresponding to the light grey region of Fig. 3 (Schwarzschild-like case) 


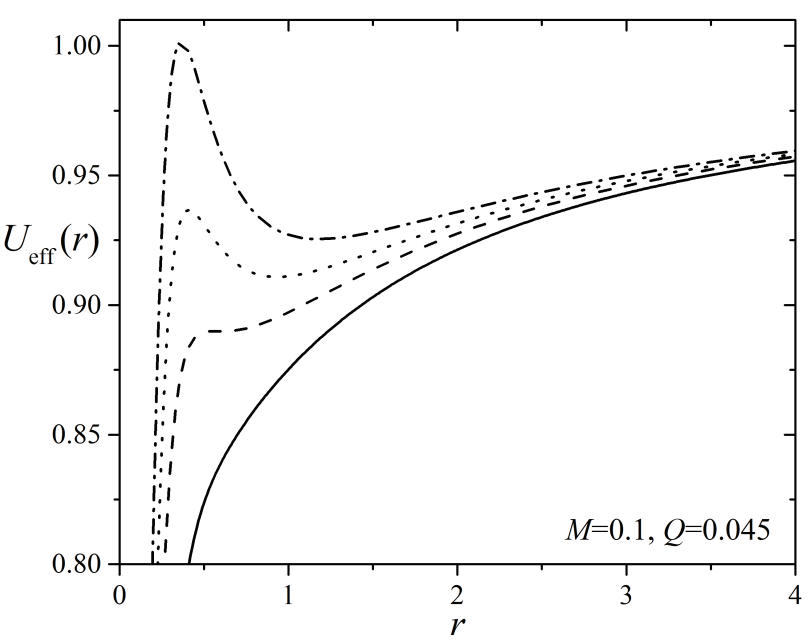

Fig. 6. Examples of $U_{\mathrm{eff}}(r, L)$ for different $L$ in the case of a configuration with a Schwarzschild-like distribution of SCOs

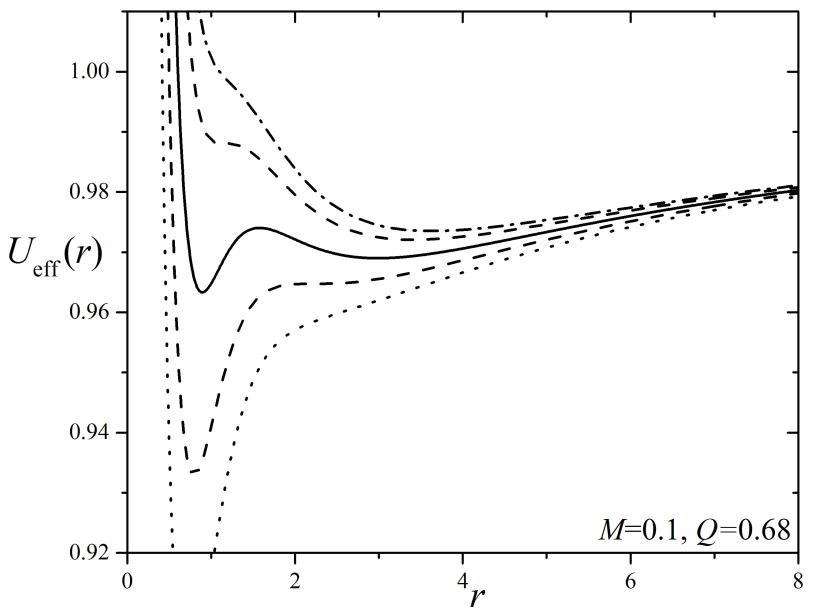

Fig. 7. Examples of $U_{\text {eff }}(r, L)$ for $L \neq 0$ in the case of a configuration where there exists separated ring-like structures of SCOs. The left minima form the inner ring of SCOs, which is separated by the unstable circular orbits (maxima of $U_{\text {eff }}$ ) from the outer SCOs

double minima of $U_{\text {eff }}$ become possible, by leading to different distributions of SCOs. The areas of $(Q, M)$ with different distributions of SCOs are separated by a bifurcation curves: when we change $(Q, M)$ so that the minima and maxima of $U_{\text {eff }}$ can appear/disappear, we have

$d F / d r=0$

for some $r$. This equation defines a curve in the plane of the parameters $(Q, M)$, which is a boundary of

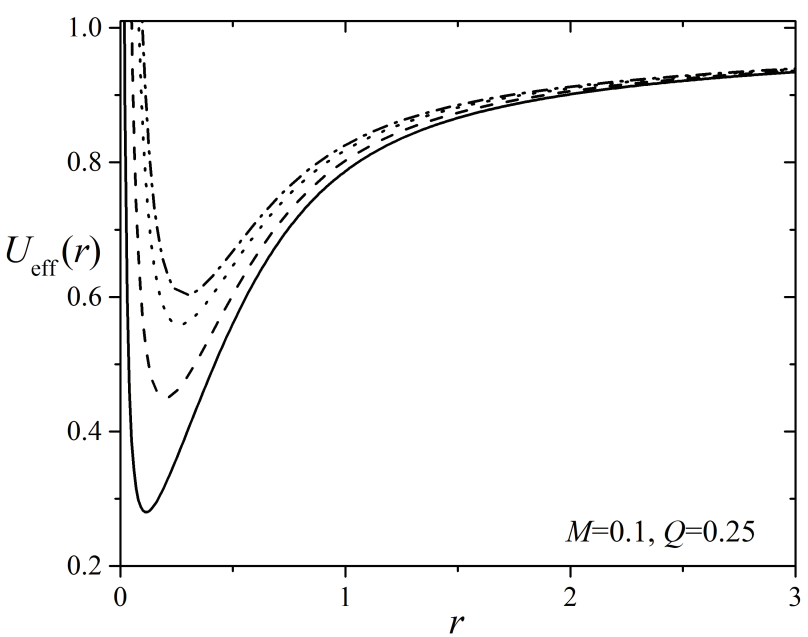

Fig. 8. Examples of $U_{\text {eff }}(r, L)$ for $L \neq 0$ in the case of a configuration where only stable SCOs can exist (dark grey region)

some parameter area in Fig. 3. From this viewpoint, the investigation of the qualitatively different distribution of SCOs reduces to the analysis of (30). Having in mind that this is a necessary (not sufficient) condition, we supplement this analysis by a direct check of the form of $U_{\text {eff }}$.

The results are presented in Fig. 3; the examples of $F(r)$ and $U_{\text {eff }}$ are shown in Figs. 4, 5, 6, 7, and 8. Possible distributions of SCOs are as follows.

(i) The Schwarzschild-like case (light grey area of the parameters in Fig. 3). There is the inner ring of unstable circular orbits. The outer ring of SCOs extends to infinity. Typical $U_{\text {eff }}$ are shown in Fig. 6 .

(ii) There are configurations, where all circular orbits are stable (dark grey area of the parameters in Fig. 3). The effective potentials for the test body trajectories have only one minimum (Fig. 8).

(iii) White area in Fig. 3 corresponds to the configurations, for which there are two rings of SCOs separated by a ring with unstable circular orbits. The outer ring of SCOs extends to infinity. In this case, $F(r)$ is non-monotonous (two upper curves in Fig. 4). The typical potential (with different $L$ ) for such a configuration is shown in Fig. 7 (solid curve).

We note that the form of $U_{\text {eff }}$ for fixed $(Q, M)$ gives information not only on the position of SCOs (minima of the potential). For example, the potential maxima (see Figs. 6, 7) corresponding to unstable circular orbits (limiting cycles) split the sets of trajectories with

ISSN 2071-0194. Ukr. J. Phys. 2019. Vol. 64, No. 3 
different qualitative behaviors, which describe, for example, loops, or infinite trajectories $r(\tau)$, or finite motions of test particles. It is worth to note that, as can be seen from Fig. 3, all types of these distributions are possible for sufficiently small $Q$ and, therefore, for sufficiently small SF.

\section{Discussion}

We investigated the circular orbits of test bodies around static spherically symmetric configurations with linear massive scalar fields. With this aim, the numerical solutions of the system of the Einstein SF equations have been obtained under conditions of the asymptotic flatness of the space-time and the zero field at the spatial infinity. For a fixed SF mass $\mu_{f}$, there are two parameters that describe the configuration: the total mass $M$ and the parameter $Q$, which defines the field strength. For given $M, Q$, we studied the distribution of stable circular orbits, using the effective potential $U_{\text {eff }}$ that describes the test body motion around the configuration.

We found that three types of distributions of SCOs are possible in the case of different $M, Q$ :

(i) the Schwarzschild-like case where there is an infinite region of SCOs, and their radii are bounded from below by the unstable orbits;

(ii) the case where all circular orbits are stable, and

(iii) there is the additional inner ring of SCOs, which is separated from the outer (infinite) ring of SCOs by a region of unstable orbits.

The numerical analysis shows that all three cases seem to survive for small values of $Q$, i.e., in the limit of small SF. In our opinion, it is of high importance that, for some choice of the configuration parameters $M, Q$, there exist distributions of SCOs (iii) in the form of two disjoint rings. In this case, the distribution of SCOs is qualitatively different from (i) and (ii). It is significant that, for most $M, Q$ of (iii) (except those corresponding to the bifurcation curves in Fig. 3), this feature is stable with respect to small changes of the parameters. The existence/nonexistence of (iii) may be used for testing the models of relativistic astrophysical objects. If such configurations with $\mathrm{SF}$ really exist, the above results on the existence of the ring structures give us a hint about the structure of thin accretion discs, which will manifest themselves in the form of relativistic lines [33,34] in the X-ray spectra.

ISSN 2071-0194. Ukr. J. Phys. 2019. Vol. 64, No. 3
This work has been supported by the scientific program "Astronomy and space physics" (project 19БФ023-01) of Taras Shevchenko National University of Kyiv.

1. B. Novosyadlyi, V. Pelykh, Yu. Shtanov, A. Zhuk. Dark Energy and Dark Matter of the Universe. Ed. by V. Shulga (Kiev, Akademperiodyka, 2013), Vol. 1 [ISBN: 978-966360-240-0].

2. A. Linde. Inflationary Cosmology after Planck 2013. (2014) arXiv:1402.0526 [hep-th].

3. S. Nojiri, S.D. Odintsov. Unified cosmic history in modified gravity: From $F(R)$ theory to Lorentz non-invariant models. Phys. Rept. 505, 59 (2011).

4. S. Nojiri, S.D. Odintsov, V.K. Oikonomou. Modified gravity theories on a nutshell: Inflation, bounce and late-time evolution. Phys. Rept. 692, 1 (2017).

5. V. Sahni. Dark matter and dark energy. Lect. Notes Phys. 653, 141 (2004).

6. E.J. Copeland, M. Sami, S. Tsujikawa. Dynamics of dark energy. Int. J. Mod. Phys. D 15, 1753 (2006).

7. K. Bamba, S. Capozziello, S. Nojiri, S.D. Odintsov. Dark energy cosmology: the equivalent description via different theoretical models and cosmography tests. Astrophys. Space Sci. 342, 155 (2012).

8. O. Sergijenko, R. Durrer, B. Novosyadlyj. Observational constraints on scalar field models of dark energy with barotropic equation of state. JCAP 08, 004 (2011).

9. B. Novosyadlyj, O. Sergijenko, R. Durrer, V. Pelykh. Do the cosmological observational data prefer phantom dark energy? Phys. Rev. D 86, 083008 (2012).

10. O. Sergijenko, B. Novosyadlyj. Sound speed of scalar field dark energy: weak effects and large uncertainties. Phys. Rev. D 91, 083007 (2015).

11. L.L. Jenkovszky, V.I. Zhdanov, E.J. Stukalo. Cosmological model with variable vacuum pressure. Phys. Rev. D 90, 023529 (2014).

12. V.I. Zhdanov, S.S. Dylda. Scalar field versus hydrodynamic models in homogeneous isotropic cosmology. Phys. Rev. D 97, Is. 12, 124033 (2018).

13. A. Diez-Tejedor. Note on scalars, perfect fluids, constrained field theories, and all that. Phys. Lett. B 727, 27 (2013).

14. G.H. Derrick. Comments on nonlinear wave equations as models for elementary particles.J. Math. Phys. 5, 1252 (1964).

15. I.Z. Fisher. Scalar mesostatic field with regard for gravitational effects. Zh. Exp. Theor. Phys. 18, 636 (1948) (in Russian).

16. A.I. Janis, E.T. Newman, J. Winicour. Reality of the Schwarzschild singularity. Phys. Rev. Lett. 20, (1968).

17. J.D. Bekenstein. Nonexistence of baryon number for static black holes. Phys. Rev. D 5, 1239 (1972).

18. J.D. Bekenstein. Black holes: classical properties, thermodynamics and heuristic quantization. In: Cosmology and Gravitation, M. Novello, ed. (Atlantisciences, 2000). 
19. R.A. Asanov. Point source of massive scalar field in gravitational theory. Teor. Matem. Fiz. 20, 1 (1974) (in Russian).

20. G. Stephenson. A static spherically symmetric solution of the Einstein-Maxwell-Yukawa field equations. Proceed. Cambridge Phil. Soc. 58(3), 521 (1962).

21. D. Solovyev, A. Tsirulev. General properties and exact models of static self-gravitating scalar field configurations. Classic. Quant. Grav. 29, 055013, (2012).

22. Z. Stuchlik, J. Schee. Appearance of Keplerian discs orbiting Kerr superspinars. Classic. Quant. Grav. 27, 215017 (2010).

23. A.N. Chowdhury, M. Patil, D. Malafarina, P.S. Joshi. Circular geodesics and accretion disks in the Janis-NewmanWinicour and gamma metric spacetimes. Phys. Rev. D 85, 104031 (2012).

24. R.S.S. Vieira, J. Schee, W. Kluźniak, Z. Stuchlík, M. Abramowicz. Circular geodesics of naked singularities in the Kehagias-Sfetsos metric of Horava's gravity. Phys. Rev. D 99, 024035 (2014).

25. D. Pugliese, H. Quevedo, R. Ruffini. Equatorial circular motion in Kerr spacetime. Phys. Rev. D 84, 044030 (2011).

26. D. Pugliese, H. Quevedo, R. Ruffini. Equatorial circular motion in Kerr spacetime. Phys. Rev. D 88, 024042 (2013).

27. D. Pugliese, H. Quevedo, R. Ruffini. General classification of charged test particle circular orbits in ReissnerNordstrem spacetime Eur. Phys. J. C 77, 206 (2017).

28. K. Boshkayev, E. Gasperin, A.C. Gutierrez-Pineres, H. Quevedo, S. Toktarbay. Motion of test particles in the field of a naked singularity. Phys. Rev. D 93, 024024 (2016).

29. V.I. Zhdanov, O.S. Stashko. The body motion in gravitational field of a spherically symmetric configuration with scalar field in General relativity. Bulletin of Taras Shevchenko National University of Kyiv, 56, 35 (2017).

30. O.S. Stashko, V.I. Zhdanov. Disconnected regions of stable circular orbits in presence of massive scalar field. Odessa Astron. Public. 30, 48 (2017).
31. O.S. Stashko, V.I. Zhdanov. Spherically symmetric configurations of General Relativity in presence of scalar fields: separation of circular orbits. Gener. Relat. Gravit. 50, 105 (2018).

32. L.D. Landau, E.M. Lifshitz. The Classical Theory of Fields: Vol. 2 (Course of Theoretical Physics) (Elsevier, 1987) [ISBN: 978-0750627689].

33. A.P. Lightman, T.R. White. Effects of cold matter in active galactic nuclei - A broad hump in the X-ray spectra. Astrophys. J. 335, 57 (1988).

34. A.C. Fabian, M.J. Rees, L. Stella, N.E. White. X-ray fluorescence from the inner disc in Cygnus X-1. Mon. Notic. Roy. Astron. Soc. 238, 729 (1989).

Received 03.02.18

О.С. Сташко, В.І. ЖКданов

СФЕРИЧНО-СИМЕТРИЧНІ КОНФІГУРАЦЇ̈

У ЗТВ У ПРИСУТНОСТІ ЛІНІЙНОГО МАСИВНОГО

СКАЛЯРНОГО ПОЛЯ: РОЗДІЛЕННЯ РОЗПОДІЛУ

КОЛОВИХ ОРБІТ ПРОБНИХ ТІЛ

$\mathrm{P}$ е $з$ ю м е

Досліджено статичні сферично-симетричні конфігурації в присутності лінійних масивних скалярних полів в 3ТВ. Розглянуто статичні асимптотично-плоскі розв'язки рівнянь Ейнштейна. Кожний розв'язок фіксується масою конфігурації і параметром, що характеризує інтенсивність поля, які визначаються на просторовій нескінченності. Метричні коефіцієнти і вигляд скалярного поля для конкретної конфігурації отримано чисельно; на цій основі досліджено часоподібні геодезичні, що описують рух пробних частинок. Основна увага приділена розподілу стійких колових орбіт (CKO) пробних частинок навколо конфігурації. Знайдено, що для множини параметрів конфігурації існують дві незв'язні області СКО, які розділені кільцевою областю, де $\mathrm{CKO}$ не існують. 